\title{
Tracking Comets and Asteroids Using Machine Learning and Deep Learning: A Review
}

\author{
M. Khalil ${ }^{1}$ *, M. Said ${ }^{1}$, A. Ibrahim ${ }^{1}$, M. Gamal ${ }^{1}$, M. A. Mobarak ${ }^{1}$ \\ ${ }^{1}$ Faculty of Engineering, October University for Modern Sciences and Arts (MSA), Giza, Egypt \\ *Corresponding author E-mail: mkibrahim@msa.eun.eg
}

\begin{abstract}
Comets and asteroids are considered as very dangerous objects to Earth. Machine Learning and Deep Learning tools can be used to identify and track such hazardous objects early enough prior to impact. Preparation strategies require several years, so the early detection of such hazardous objects is very crucial aspect. In this paper, the importance of using Machine Learning and Deep Learning to identify and detect asteroids and comets is discussed briefly.
\end{abstract}

Keywords: Machine Learning; Deep Learning; Tracking of Comets and Asteroids; Long Period Comets.

\section{Introduction}

Recently, Machine Learning and Deep Learning have become the most popular techniques in astronomy which can be used to extract useful information and knowledge from Big Data [2]. Using Machine Learning and Deep Learning helps researchers to identify and track asteroids and comets [3-5]. The aim of this paper is to survey and exaggerate the fundamentality of such approaches, mainly the usage of Machine Learning and Deep Learning in astronomical identifications. They both do operate on the same principle, on can say though that Deep-Learning (DL) is more complicated compact form of Machine-Learning (ML), however, they both do operate on the same principle, mainly, a perceptron $[6,8]$. Let $\hat{\mathrm{f}}(\mathrm{x})$ be a vector valued function, such that

$\hat{f}(x)=\varphi(\omega \cdot x+b)$ whereas, $\varphi(\alpha)= \begin{cases}1, & \alpha>0 \\ 0, & \text { otherwise }\end{cases}$

This is called an activation function, it is a simple function that would only work if and only if a condition is met. The ecstasy lies in defining such variables/parameters in which will provide the best fit to our data, this is called training. Neural networks are a punch of these perceptrons with inputs, outputs and weights, these all with the activation function plays the role of providing or predicting data, based on the training set, or the given set. Some methods were introduced to best fit the result by the means of adjusting the weights like gradient descent; $\theta^{*}=\theta-[n * \nabla J(y, x ; \theta)]$, in where $\theta^{*}, \theta$ and $\mathrm{n}$ are the new weight, the old weight, and the learning rate respectively, $\mathrm{J}$ is the gradient. Some forms like backpropagation and principal component analysis (PCA) do offer such means as well. A collection of layers, hidden ones, result in Deep Learning, while a simpler form would be a Machine Learning $[6,8]$.

Machine Learning (ML) is considered a subset of AI techniques that allows computer systems to be taught from past experiences for example "data observations" and improve their responses for a given task. ML techniques include Support Vector Machines (SVM), decision trees, Bayes learning, k-means clustering, association rule learning, regression, neural networks, and much more. Where Deep Learning (DL) is considered to be a subset of Neural networks that allows the computational multi-layered to make NN more attainable. normal DL structures are deep neural networks (DNNs), convolutional neural networks (CNNs), recurrent neural networks (RNNs), generative adversarial networks (GAN), and a lot more. Applying one of these approaches to a given data can take a huge amount of time, based on the computing power and storage abelites that are available for scientists. As soon as our data is prepared ones should choose the best machine learning or deep learning algorithm for solving the problem especially when facing a huge amount of data, we should start asking few questions like which the best algorithm is to be considered as a solution for our problem, it depends on many factors such as:

- Quality.

- $\quad$ Size.

- Time available for computing.

- $\quad$ The desired prediction and loss. 


\section{Identifying asteroids and comets by means of deep learning}

By artificial neural networks, this technology can detect asteroids that can affect Earth. The resulting of trained AI system instrument named the Hazardous Object Identifier (HOI), was trained based on an artificial group of known effects "that produced by launching objects from Earth's surface after that integrating these objects from Earth's with backward in time. HOI could identify $95.25 \%$ of the known asteroids affected of simulation system that were produced in the test set as potential effects. In other way, could detect near to 90.99\% of the probable hazardous asteroids detected by NASA, without having a direct training. Neural networks more flexible to disarranged modes of the initial conditions for chaotic motion. To know the network's accuracy, it is based on cross-entropy law: $H(y, \hat{y})=-\sum_{i}^{N} y_{i} \ln \left(\hat{y}_{i}\right)+\left(1-y_{i}\right) \ln \left(1-\hat{y}_{i}\right)$. Here $\mathrm{y}$ is the actual value, or label, $\hat{\mathrm{y}}$ is the predicted value, and $\mathrm{N}$ is the total amount of predictions. This cost function has the convenient property that its derivative with respect to some input weight w, scales linearly with the difference between the label and predicted value $\frac{d C}{d w}=\frac{1}{N} \sum_{i}^{N} x\left(\hat{y}_{i}-y_{i}\right)$. One other use if CAMS, short for Cameras for All Sky Meteor Surveillance. It is a data analysis tube to classify meteors from other detect types $[6,8]$. This system helps to processing the data that is collected every day from low-light videos surveillance and makes results available to the monitoring detections. By the usage of DL in from of a CNN (Convolutional Neural Network), and a Long-Term Memory Network (LSTM), this system performance makes his models appropriate to be deployed on site and help relieve the difference load on astronomers to classify meteors or non-meteors. The LSTM receive curves for light tracks into a space, and train to predict meteors or non-meteors. The system used one disappeared layer and classifier software. it used cross-validation to measure the number of disappeared units. The CNN took a group of inputs of pixel image frames after that whereas the LSTM took values of the observed time-series light power. These methods allow system to provide clean data to identify meteoroid or not meteoroid by using orbits potentially related to long-period comets that pass near to Earth's orbits.

\section{Searching for long-period comets with deep learning tools}

Long-period comets are recognized as the potentially most devastating threat to Earth due to their large size and fast impact speeds of up to $72 \mathrm{~km} / \mathrm{s}$ along with short-period comets and asteroids [3,4]. Any new long-period comet can be discovered 6-12 months before impact. To provide extra warning time, Deep Learning is applied in order to fully automate meteor shower detection and classification from video observations and to provide comet searchers directions on where to look for comets when they are still far out. So it would be possible to avoid the impact by altering the asteroid's orbit through a gravitational tugboat [7] or by striking it with a nuclear weapon. Both strategies require many years of preparation, which makes the early detection of such hazardous objects vital.

\section{Conclusions and recommendations}

In this work, we present a detailed overview of using Machine learning and Deep Learning tools in identifying and tracking asteroids and comets which are the most potentially hazardous objects to Earth. Such tools can provide extra warning time. We encourage and motivate researchers to share and study the gathered data from low-light video observations in order to obtain a better understanding of the behaviour of such hazardous objects.

\section{References}

[1] Khalil, M., Said, M., Osman, H., Ahmed, B., Ahmed, D., Younis, N., Maher, B., Osama, M. and Ashmawy, M., Big data in astronomy: from evolution to revolution, International Journal of Advanced Astronomy, 7 (1) (2019) 11-14. https://doi.org/10.14419/ijaa.v7i1.18029.

[2] Khalil, M., Said, M., Osman, H., Younis, N., Khaled N., Amr, Y., Mohamed, N.A., Ibrahim, A., 2020. Dark comets: the cosmic catastrophic threat to earth, 8 (1) (2020) 27-28. https://doi.org/10.14419/ijaa.v8i1.30740.

[3] Khalil, M., Said, M., Osman, H., Younis, N., Khaled N., Amr, Y., Mohamed, N.A., Ibrahim, A., 2020. The Dazzling Comet C/2020 F3 (NEOWISE): The Comet of The Century, 8 (2) (2020) 35-36. https://doi.org/10.14419/ijaa.v8i2.31149.

[4] Khalil, M., Said, M., Osman, H., Younis, N., Khaled N., Amr, Y., Mohamed, N.A., Ibrahim, A., 2021, 'Oumuamua: A Mysterious Visitor from Deep Space, 9(1) 24-25.

[5] Milani, A., Chesley, S.R., Chodas, P.W. and Valsecchi, G.B., 2002. Asteroid close approaches: analysis and potential impact detection. Asteroids III, 1, pp.89-101.

[6] Schweickart, R.L., Lu, E.T., Hut, P. and Chapman, C.R., 2003. The asteroid tugboat. Scientific American, 289(5), pp.54-61. https://doi.org/10.1038/scientificamerican1103-54.

[7] Zoghbi, S., Cicco, M., Stapper, A.P., Ordonez, A.J., Collison, J., Gural, P.S., Ganju, S., Galache, J.L. and Jenniskens, P., 2017. Searching for longperiod comets with deep learning tools. In Deep Learning for Physical Science Workshop, NeurIPS. 